\title{
A Linguistic Multi-Criteria Decision Making Model Applied to the Integration of Education Questionnaires
}

\author{
Ramón A. Carrasco, Pedro Villar, Miguel J. Hornos \\ Department of Software Engineering, University of Granada, \\ Granada 18071, Spain \\ E-mail: $\{$ racg, pvillarc, mhornos\}@ugr.es \\ Enrique Herrera-Viedma \\ Department of Computer Science and Artificial Intelligence, University of Granada, \\ Granada 18071, Spain \\ E-mail:viedma@decsai.ugr.es \\ Received 6 April 2011 \\ Accepted 29 September 2011
}

\begin{abstract}
We present a model made up of linguistic multi-criteria decision making processes to integrate the answers to heterogeneous questionnaires, based on a five-point Likert scale, into a unique form rooted in the widespread course experience questionnaire. The main advantage of having the resulting integrated questionnaire is that it can be incorporated into other course experience questionnaire surveys to make benchmarking among organizations. This model has been applied to integrate heterogeneous educational questionnaires at the University of Granada.
\end{abstract}

Keywords: Linguistic multi-criteria decision making, Integration of questionnaires, Education questionnaires, Course experience questionnaire.

\section{Introduction}

Interest in the nature of the university students' experience in most countries has grown considerably in recent years. Studies of student life have also grown at the institutional level in response to quality assurance demands. Partly in anticipation of external pressures to provide evidence of student satisfaction, and partly to anticipate and deflect criticism, many universities are generating quite sophisticated studies of student life to inform their policy and planning. Until recently, this has been more prominent in Anglophone countries, but now has become an almost inescapable feature of university strategic planning initiatives in most developed systems. ${ }^{1}$ The vast majority of these studies are based on questionnaires answered by the students. There are substantial differences in the focus of national preoccupations about student life, even among different universities and/or university colleges in the same country. Therefore, these questionnaires are generally very heterogeneous.

However, there are some emerging methods of survey that try to be more general. Thus, the national survey of student engagement (NSSE) ${ }^{2}$ obtains, on an annual basis, information from North-American universities and colleges about student participation and perceptions on good educational practices. The course experience questionnaire (CEQ) ${ }^{3-6}$ is used in many Anglo-Saxon universities around the world (mainly in Australia and the United Kingdom). CEQ is a long-term theory and research into the student learning experience which forms the basis of performance indicators. The 
basic form of CEQ is made up of 24 statements (questions) about five prescribed areas (called scales) of the courses: good teaching, generic skills, student support, graduate qualities and overall satisfaction. This form asks students to agree or disagree for each question on a Likert scale.

Information gathered in education questionnaires often involves the use of such Likert-type scales. ${ }^{7}$ The Likert scale invention is attributed to Rensis Likert, ${ }^{8}$ who described this technique for the assessment of attitudes. Ref. 9 describes the Likert scale as a set of items, made up of approximately an equal number of favorable and unfavorable statements concerning the attitude object, which is given to a group of subjects. They are asked to respond to each statement in terms of their own degree of agreement or disagreement. Typically, they are instructed to select one of five responses (five-point Liker scale): "strongly agree", "agree", "neutral", "disagree", or "strongly disagree". In general, these human perceptions are characterized by uncertainty and fuzziness, ${ }^{10}$ i.e. they are subjective and vague. Furthermore, variations in individual perceptions and personality mean that the same words can indicate very different perceptions. ${ }^{11}$ Consequently, the use of binary logic and crisp numbers to describe these human perceptions or attitudes (e.g. "strongly agree" $=2$, "agree" $=1$, "neutral" $=0$, "disagree" $=-1$, and "strongly disagree" $=-2$ ) fails to address fuzziness. ${ }^{12}$ In that case, a better approach should be based on the use of linguistic assessments instead of numerical values. The fuzzy linguistic approach was introduced by Zadeh in $1975 .{ }^{13}$ It is a tool intended to model qualitative information that has been used successfully on many domains and problems. ${ }^{14-23}$ This approach is based on the concept of linguistic variable. Briefly speaking, linguistic variables are variables whose values are not numbers, but words or sentences in a natural or artificial language. Therefore, the fuzzy linguistic approach seems to be an appropriate framework for modeling the information of questionnaires like the ones in which the Likert scale is used.

The CEQ form asks students to agree or disagree on a five-point Likert scale. Moreover, many other different questionnaires use this type of scale and deal with the same areas mentioned above for CEQ. Although these questionnaires have been carried out on different university populations, they may have one or several common features (dimensions), e.g. time (academic year, semester,...), space (University College, region of the university,...), etc. However, the contents of these questionnaires are usually very heterogeneous. The main aim of this paper is to integrate such heterogeneous questionnaires, in order to obtain a unique CEQ form with the integrated answers to the input questionnaires. This new integrated questionnaire will characterize the input questionnaires on their common features. The main advantage of having this integrated questionnaire is that it can be incorporated into other CEQ surveys in order to make benchmarking among organizations.

The computation model to generate the integrated questionnaire has been approached as two linguistic multi-criteria decision making (LMCDM) processes. ${ }^{11,14-16}$ The goal of such processes consists of searching the best alternatives according to the linguistic assessments provided by a group of users with respect to a set of evaluation criteria. The respective aims of the two LMCDM processes are:

(i) To associate each question of the input questionnaires with a linguistic value indicating how is the matching of that question with each of the previously mentioned conceptual CEQ scales. In this step, it is necessary the consensus of a group of university education experts.

(ii) To obtain a unique CEQ-type form with the integrated answers to the input questionnaires. This integration process will be weighted with the linguistic values obtained in the previous step. In this step, the decision makers are the students who have filled in the corresponding questionnaires.

Several web questionnaires for evaluating different questions have been used during the last academic years to survey the students enrolled on Information and Communication Technologies (ICT) courses taught at the Business Studies Faculty of the University of Granada. The new model presented in this paper has been applied for integrating these heterogeneous questionnaires into a unique CEQ-type form. In this paper, we show a limited example of this integration problem.

The rest of the paper is organized as follows: Section 2 revises the preliminaries concepts, i.e. the methods for solving decision making problems under linguistic information (opinions). Section 3 explains the CEQ form in more details. Section 4 presents the new linguistic integration model. Section 5 shows an 
application example of this model. Finally, we point out some concluding remarks and future work.

\section{Solving Decision Making Problems under Linguistic Information}

The main aim of this section is to present a study on the steps to follow in linguistic decision analysis, that is, the steps to follow for solving a decision making problem under linguistic information. In all real-world decision making processes, there are usually various actors (experts or decision makers) who are called to express their performance values on a predefined set of options (alternatives) in order to select the best one(s). We do not distinguish between "experts" and "criteria", and interpret linguistic decision analysis in a context of multi-criteria decision making (MCDM). ${ }^{11,14-16}$ In the linguistic decision analysis of an MCDM problem, the solution scheme must be formed by the following three steps: ${ }^{16}$ the choice of the linguistic term set with its semantics, the choice of the aggregation operator of linguistic information, and the choice of the best alternatives. In the following subsections, we analyze these phases.

\subsection{Choosing the Linguistic Term Set with its Semantics}

The choice of the linguistic term set with its semantics is the first goal to fulfill in any linguistic approach for solving a problem. It consists of establishing the linguistic variable ${ }^{13}$ or linguistic expression domain with a view to provide the linguistic performance values. Since the concept was introduced, ${ }^{13}$ linguistic variables have been widely used. Briefly speaking, linguistic variables are variables whose values are not numbers, but words or sentences in a natural or artificial language; and these values of linguistic variables are called linguistic labels. In more specific terms, a linguistic variable is characterized by a quintuple $\langle H, T(H), U, G, M\rangle$, where:

- $H$ is the name of the variable.

- $T(H)$ is the term-set of $H$ or the collection of linguistic values (labels).

- $U$ is the universe of discourse.

- $G$ is the syntactic rule, i.e. a context-free grammar which generates the terms in $T(H)$.

- $M$ is the semantic rule which defines the meaning of each linguistic label $X, M(X)$, where $M(X)$ denotes a fuzzy subset of $U$.
The fuzzy linguistic approach ${ }^{13}$ is a tool intended for modeling qualitative information in a problem. It is based on the concept of linguistic variable and has been satisfactorily used in many domains, ${ }^{17-20}$ including decision-making problems. $^{21-23}$ The main aim of establishing the linguistic descriptors of a linguistic variable is to supply the user with a few words by which $\mathrm{s} /$ he can naturally express her/his information. In order to accomplish this objective, an important aspect to analyze is the "granularity of uncertainty", i.e. the level of discrimination among different counts of uncertainty. Typical values of cardinality used in the linguistic models are odd ones, such as 5 or 7 , where the mid term represents an assessment of "approximately 0.5 ", and with the rest of the terms being placed symmetrically around it. ${ }^{24}$ Once the cardinality of the linguistic term set has been established, the linguistic terms and their semantics must be provided:

(i) Generation of the linguistic terms. Mainly, there are two possibilities to accomplish this task..$^{17,25-27}$ One of them involves directly supplying the term set by considering all the terms distributed on a scale on which a total order is defined. ${ }^{26,27}$ The other specifies a context-free grammar. ${ }^{17}$

(ii) Semantics of the linguistic terms. Often, the semantics of the terms are represented by fuzzy numbers, defined in the interval $[0,1]$, described by membership functions. A way to characterize a fuzzy number is to use a representation based on parameters of its membership function. ${ }^{24}$ The linguistic assessments given by the users are just approximate ones. Some authors consider that linear trapezoidal membership functions are good enough to capture the vagueness of such linguistic assessments. $^{21}$ The parametric representation is achieved by the 4-tuple $\langle\alpha, \beta, \gamma, \delta\rangle$, where $\beta$ and $\gamma$ indicate the interval in which the membership value is 1 , with $\alpha$ and $\delta$ indicating the left and right limits of the definition domain of the trapezoidal membership function. ${ }^{24}$ A particular case of this type of representation are the linguistic assessments whose membership functions are triangular, i.e. $\beta=$ $\gamma$. Some authors ${ }^{28}$ introduce a modifier that leads to a decrease or an increase in the degrees of membership. Furthermore, the authors of ordinal linguistic models to establish the semantics of the linguistic terms consider that each linguistic term for the pair $\left(s_{i}, s_{T-i}\right)(T+1$ is the cardinality of the set $S$ of linguistic terms) is equally informative function. ${ }^{16}$ 


\subsection{Choosing the Linguistic Information Aggregation Operator}

There are two main approaches for carrying out the aggregation of linguistic information:

- The approximative computational model based on the Extension Principle. ${ }^{24}$ This model uses fuzzy arithmetic based on the Extension Principle to make computations over the linguistic variables. The results can be presented in two ways: by means of the fuzzy numbers obtained from the fuzzy arithmetic computations based on the Extension Principle; or by means of linguistic labels computed from the fuzzy numbers obtained by performing a linguistic approximation process.

- The ordinal linguistic computational model..$^{29,30}$ This symbolic model makes direct computations on labels, using the ordinal structure of the linguistic term set $S=\left\{s_{0}, \ldots, s_{T}\right\}$. Its results are inherently linguistic labels, due to either the operators used (basically negation, maximization and minimization operators) or because in the computations on the order index there exist an approximation by means of the round operator.

In the literature, we can find four kinds of aggregation operators of linguistic information: non-weighted linguistic information aggregation operators, ${ }^{30}$ weighted linguistic information aggregation operators, ${ }^{31}$ multigranularity linguistic information aggregation operators, ${ }^{15,21}$ and numeric and linguistic information aggregation operators ${ }^{16}$. In the following subsections, we review the kinds of linguistic information aggregation operators based on symbolic computation that we use in our model.

\subsubsection{LOWA Operator}

An important aggregation operator of ordinal linguistic values based on symbolic computation is the Linguistic Ordered Weighted Averaging (LOWA) operator, ${ }^{30}$ which is used to aggregate non-weighted ordinal linguistic information, i.e. linguistic information values with equal importance.

Definition 2.1. Let $A=\left\{a_{1}, \ldots, a_{m}\right\}$ be a set of labels to be aggregated, then the LOWA operator, $\varphi$, is defined as:

$$
\begin{aligned}
& \varphi\left(a_{1}, \ldots, a_{m}\right)=W \cdot B^{T}=C^{m}\left\{w_{k}, b_{k}, k=1, \ldots, m\right\} \\
= & w_{1} \otimes b_{1} \oplus\left(1-w_{1}\right) \otimes C^{m-1}\left\{\beta_{h}, b_{h}, h=2, \ldots, m\right\},
\end{aligned}
$$

where $W=\left[w_{1}, \ldots, w_{m}\right]$ is a weighting vector, such that $w_{i} \in[0,1]$ and $\sum_{i=1}^{m} w_{i}=1, \beta_{h}=w_{h} / \sum_{k=2}^{m} w_{k}$, and $B=$

$\left\{b_{1}, \ldots, b_{m}\right\}$ is a vector associated with $A$, such that $B=$ $\sigma(A)=\left\{a_{\sigma(1)}, \ldots, a_{\sigma(m)}\right\}$, where $a_{\sigma(j)} \leq a_{\sigma(i)}, \forall i \leq j$, with $\sigma$ being a permutation over the set of labels $A . C^{m}$ is the convex combination operator of $m$ labels and if $m=2$, then it is defined as:

$$
C^{2}\left\{w_{i}, b_{i}, i=1,2\right\}=w_{1} \otimes s_{j} \oplus\left(1-w_{1}\right) \otimes s_{i}=s_{k},
$$

such that $k=\min \left\{T, i+\operatorname{round}\left(w_{1} \cdot(j-i)\right)\right\}, s_{j}, s_{i} \in S,(j \geq$ $i$ ), where round is the usual round operation, and $b_{1}=s_{j}$, $b_{2}=s_{i}$. If $w_{j}=1$ and $w_{i}=0$, with $i \neq j, \forall i$, then the convex combination is defined as $C^{m}\left\{w_{i}, b_{i}, i=1, \ldots, m\right\}$ $=b_{j}$.

The LOWA operator is an or-and operator ${ }^{30}$ and its behavior can be controlled by means of $W$. In order to classify OWA operators with regards to their localization between or and and, Yager ${ }^{32}$ introduced a measure of orness, associated with any vector $W$ :

$$
\operatorname{orness}(W)=\frac{1}{m-1} \sum_{i=1}^{m}(m-i) w_{i}
$$

This measure characterizes the degree to which the aggregation is like an or ( $\max )$ operation. Note that an OWA operator with orness $(W) \geq 0.5$ will be an orlike, and with orness $(W)<0.5$ will be an andlike operator.

An important question of the LOWA operator is the determination of the weighting vector $W$. In Ref. 32, it was defined an expression to obtain $W$ that allows representing the concept of fuzzy majority ${ }^{33}$ by means of a fuzzy linguistic non-decreasing quantifier $Q:{ }^{34}$

$$
w_{i}=Q(i / n)-Q((i-1) / n), i=1, \ldots, n .
$$

When a fuzzy linguistic quantifier $Q$ is used to compute the weights of the LOWA operator $\varphi$, it is symbolized by $\varphi_{Q}$.

\subsubsection{LWA Operator}

Another important aggregation operator of ordinal linguistic values is the Linguistic Weighted Averaging (LWA) operator. $^{32}$ It is based on the LOWA operator and is defined to aggregate weighted ordinal fuzzy linguistic information, i.e. linguistic information values with not equal importance.

As it is known, the aggregation of weighted information involves two activities: the transformation of the weighted information under the importance 
degrees by means of a transformation function $h$, and the aggregation of the transformed weighted information by means of a non-weighted information aggregation operator $f$. The transformation function depends on the type of weighted information aggregation which is going to be performed. In Ref. 32, Yager discussed the effect of the importance degrees in the max and min types of aggregation and suggested a class of functions for importance transformation in both types of aggregation. For the $\min$ aggregation, he suggested a family of $t$-conorms acting on the weighted information and the negation of the importance degree, which presents the non-increasing monotonic property in these importance degrees. For the max aggregation, he suggested a non-decreasing monotonic property in these importance degrees.

Following the above idea, the LWA operator is defined in Ref. 30. Here, we redefine it to simplify its expression using the orness measure and as $f$ the LOWA operator $\varphi$.

Definition 2.2. The aggregation of a set of weighted linguistic opinions, $\left\{\left(c_{1}, a_{1}\right), \ldots,\left(c_{m}, a_{m}\right)\right\}$, with $c_{i}, a_{i} \in$ $S$, according to the LWA operator $\Phi$ is defined as:

$$
\Phi\left[\left(c_{1}, a_{1}\right), \ldots,\left(c_{m}, a_{m}\right)\right]=\varphi\left(h\left(c_{1}, a_{1}\right), \ldots, h\left(c_{m}, a_{m}\right)\right),
$$

where $a_{i}$ represents the weighted opinion, $c_{i}$ the importance degree of $a_{i}$, and $h$ is the transformation function defined depending on the weighting vector $W$ used for the LOWA operator $\varphi$, such that $h=\min \left(c_{i}, a_{i}\right)$, if $\operatorname{orness}(W) \geq 0.5$, and $h=\max \left(\operatorname{neg}\left(c_{i}\right), a_{i}\right)$, if $\operatorname{orness}(W)<0.5$.

We should point out that the LOWA and LWA operators are the basis of the LMCDM processes that we present in this paper. We have chosen these operators due to the following reasons:

- Both operators are complementary (the LWA operator is defined from the LOWA operator), and this simplifies the design of the model.

- Since both operators act by symbolic computation, linguistic approximation processes are unnecessary. Consequently, this simplifies the computing processes with words.

- The concept of fuzzy majority represented by linguistic quantifiers acts in the computation processes, in such a way that the assessments on questionnaires are obtained according to the majority of opinions provided by the users (education experts and students).

\subsection{Choosing the Best Alternatives}

Assuming a linguistic framework in an MCDM problem, we have linguistic performance values $\left\{V_{1}, \ldots\right.$, $\left.V_{m}\right\}$ about a set of alternatives $X=\left\{x_{1}, \ldots, x_{n}\right\}$ provided according to a group of criteria $\left\{P_{1}, \ldots, P_{m}\right\}$. Then, the goal consists of finding the best alternatives from the linguistic performance values. This goal is achieved by means of a choice process among the alternatives. ${ }^{35,36}$ As is known, two approaches may be considered basically to carry out a choice process. ${ }^{26,33} \mathrm{~A}$ direct approach: $\left\{V_{1}, \ldots, V_{m}\right\} \rightarrow$ the best alternatives, according to which a solution with the best alternatives is derived on the basis of the individual preferences; and an indirect approach: $\left\{V_{1}, \ldots, V_{m}\right\} \rightarrow$ the best alternatives, providing the best alternatives on the basis of a collective preference, $V^{C}$, which is a preference of the group of criteria as a whole. Here, we assume an indirect approach.

The proposed choice process is carried out in two phases: the linguistic information aggregation phase and the exploitation phase for the aggregated linguistic information. In the following subsections, we analyze both phases. ${ }^{16}$

\subsubsection{Aggregation Phase}

The goal of the aggregation phase in the linguistic decision analysis of an MCDM problem is to obtain a collective linguistic performance value $V^{C}$ from the individual ones $\left\{V_{1}, \ldots, V_{m}\right\}$ provided for the criteria, using the aggregation operator chosen in the previous step.

At the beginning of linguistic decision analysis, we should establish what kind of representation to use for providing the linguistic performance values. Traditionally, the linguistic preferences can be provided in any of these two ways: ${ }^{30}$

- Linguistic preference relation. In this case, for a criterion $k$, a linguistic preference relation is supplied over the set of alternatives $V_{k}=v_{k}^{i j}$, where each element of the relation $v_{k}^{i j}$ reflects the linguistic degree to which an alternative $x_{i}$ is preferred to another $x_{j}{ }^{26,37}$

- Linguistic utility function. In this case, for each criterion, a utility function $V_{k}=\left[v_{1}{ }^{k}, \ldots, v_{n}{ }^{k}\right]$ is supplied that associates each alternative $x_{i}$ with a linguistic value $v_{j}^{k}$, indicating the performance of that alternative. ${ }^{27,38-40}$ 
Thus, if the linguistic performance values $\left\{V_{1}, \ldots, V_{m}\right\}$ are linguistic utility functions, then $V^{C}$ will be a collective linguistic utility function, and if $\left\{V_{1}, \ldots, V_{m}\right\}$ are linguistic preference relations, then $V^{C}$ will be a collective linguistic preference relation.

\subsubsection{Exploitation Phase}

The goal of this phase is to choose the best alternatives from $V^{C}$. Usually, the exploitation is modeled using choice functions which allow us to characterize the alternatives and to separate the best alternatives. ${ }^{15,16,41,42}$ Each alternative is characterized by means of a choice degree calculated from a collective performance value, in such a way that a rank ordering among the alternatives is defined. Later, the alternatives with the maximum choice degree are chosen. Therefore, assuming a linguistic framework, the exploitation step consists of two tasks: i) obtain a rank ordering among the alternatives by means of a linguistic choice function defined from the collective linguistic performance value $V^{C}$, in such a way that a linguistic choice set of alternatives is obtained: $X^{C}=\left\{\left(x_{j}, \mu_{X} c\left(x_{j}\right)\right), j=1, \ldots, n\right\}$ and $\mu_{X} c: X \rightarrow S$; and ii) choose the best alternatives according to the established rank ordering. Here, a solution set of alternatives is obtained as follows:

$$
X^{S}=\left\{x_{i} \in X \mid \mu_{X} c\left(x_{i}\right)=\operatorname{Max}_{x \in X}\left\{\mu_{X} c\left(x_{j}\right)\right\}\right\} .
$$

The definition of a linguistic choice function depends on the type of representation chosen initially to provide the linguistic performance values. As mentioned above, if the linguistic performance values are linguistic utility functions, then $V^{C}$ will be a collective linguistic utility function, and the establishment of a rank ordering is a direct and easy process, since $V^{C}$ is itself a linguistic choice function, ${ }^{27,38-40}$ i.e. $V^{C}=X^{C}$. Then, the solution set of alternatives is obtained as follows:

$$
X^{S}=\left\{x_{i}, x_{i} \in X \mid V^{C}\left(x_{i}\right)=\operatorname{Max}_{x_{j \in X}}\left\{V^{C}\left(x_{j}\right)\right\}\right\} .
$$

However, if the linguistic performance values are linguistic preference relations, then $V^{C}$ will be a collective linguistic preference relation. In this case, the establishment of a rank ordering is not an easy and direct task; for a more detailed explanation, see Refs. 16 and 42 .

\section{Course Experience Questionnaire (CEQ)}

The basic form of CEQ was developed for initial graduates in the United Kingdom. ${ }^{3}$ A later version was tested in Australian universities during 1989. ${ }^{4}$ Since then, the Graduate Careers Council of Australia's Survey Management Group has progressively refined the contents of this instrument. The items included in the questionnaire have changed, and the wording of some items has been modified in response to data obtained from the application of the instrument in different contexts. In Ref. 6 there is a report on the validity and usefulness of CEQ as a performance indicator of the perceived quality of university teaching. The final five scales recommended for an extended CEQ are: ${ }^{5}$

(i) Good Teaching Scale (GTS) is characterized by practices such as providing students with feedback on their progress, explaining things, making the course interesting, motivating students, and understanding students' problems. High scores on GTS are associated with the perception that these practices are present. Lower scores reflect a perception that these practices are carried out less frequently. There is a body of research that links these practices to positive learning outcomes.

(ii) Generic Skills Scale (GSS) is an attempt to take into account the extent to which university courses add to the generic skills that their graduates might be expected to possess. While discipline-specific skills and knowledge are often crucial to prospects of employment and further studies, the emphasis on generic skills stems from the belief that knowledge quickly becomes obsolete, and generic skills that may have been acquired in the learning process should endure and be applicable in a broader context. Skills typically identified in this context include communication skills, the capacity to learn new skills and procedures, the capacity to make decisions and solve problems, the ability to apply knowledge to the workplace, and the capacity to work with minimum supervision.

(iii) Student Support Scale (SSS) attempts to measure access to, and satisfaction with, key university facilities and services supporting student learning outcomes.

(iv) Graduate Qualities Scale (GQS) focuses on qualities typically associated with university outcomes, especially attitudes and perspectives related to the relevance of the course for lifelong learning. 
(v) Overall Satisfaction Index (OSI) indicates students' overall satisfaction level with the course through one question: "Overall, I was satisfied with the quality of this course".

The basic form of the CEQ presents students 24 statements (questions) about the five above mentioned scales (see Table 1) to ask them if they are agree or disagree on a five-point Likert scale. characterizes them on their common features. Given the requirement to integrate the existing questionnaires, we cannot use the CEQ basic form, made up of the questions shown in Table 1, because they generally are not available in the original questionnaires. Therefore, we have to use the major conceptual CEQ scales previously explained. We have applied the following formal framework to the problem we are trying to solve:

Table 1. Basic form of the CEQ.

\begin{tabular}{|c|c|}
\hline Scale & Question \\
\hline $\begin{array}{l}\text { Good Teaching } \\
\text { Scale (GTS) }\end{array}$ & $\begin{array}{l}\text { The teaching staff of this course motivated me to do my best work. } \\
\text { The staff put in a lot of time into commenting on my work. } \\
\text { The staff made a real effort to understand difficulties I might be having with my work. } \\
\text { The teaching staff normally gave me helpful feedback on how I was going. } \\
\text { My lecturers were extremely good at explaining things. } \\
\text { The teaching staff worked hard to make their subjects interesting. }\end{array}$ \\
\hline $\begin{array}{l}\text { Generic Skills } \\
\text { Scale (GSS) }\end{array}$ & $\begin{array}{l}\text { The course developed my problem-solving skills. } \\
\text { The course sharpened my analytic skills. } \\
\text { The course helped me develop my ability to work as a team member. } \\
\text { As a result of my course, I feel confident about tackling unfamiliar problems. } \\
\text { The course improved my skills in written communication. } \\
\text { My course helped me to develop the ability to plan my own work. }\end{array}$ \\
\hline $\begin{array}{l}\text { Student Support } \\
\text { Scale (SSS) }\end{array}$ & $\begin{array}{l}\text { I was able to access information technology resources when I needed them. } \\
\text { Relevant learning resources were accessible when I needed them. } \\
\text { Health, welfare and counseling services met my requirements. } \\
\text { The library services were readily accessible. } \\
\text { I was satisfied with the course and careers advice provided. }\end{array}$ \\
\hline $\begin{array}{l}\text { Graduate } \\
\text { Qualities Scale } \\
\text { (GQS) }\end{array}$ & $\begin{array}{l}\text { The course provided me with a broad overview of my field of knowledge. } \\
\text { The course developed my confidence to investigate new ideas. } \\
\text { University stimulated my enthusiasm for further learning. } \\
\text { I learned to apply principles from this course to new situations. } \\
\text { I consider what I learned valuable for my future. } \\
\text { My university experience encouraged me to value perspectives other than my own. }\end{array}$ \\
\hline $\begin{array}{l}\text { Overall } \\
\text { Satisfaction } \\
\text { Index (OSI) }\end{array}$ & Overall, I was satisfied with the quality of this course. \\
\hline
\end{tabular}

\section{Linguistic Integration Process of Answers to Input Questionnaires into an Output CEQ Form}

In our problem, we have several questionnaires placed in different sites (generally websites), with different university-level questions. Although these questionnaires have been filled in by different university populations, they have one or several common features (dimensions), e.g. time (academic year, semester,...), space (University College, region of the university,...), etc. Besides, all the items are five-point Likert type questions. The objective is to aggregate these questionnaires into a five-point Likert CEQ form that
Let $R=\left\{R_{l}, \ldots, R_{\# R}\right\}$ be a collection of non-empty sets of university-level questions, i.e. questionnaires to be aggregated (input questionnaires), where $\# R \geq 1$ is the cardinality of the set $R$. Let each questionnaire $R_{a}=$ $\left\{r_{1}^{a}, \ldots, r_{\# R a}^{a}\right\}, \forall a \in\{1, \ldots, \# R\}, \# R_{a} \geq 1$, be a set of $\# R_{a}$ five-point Likert type questions. Let $C E Q=$ $\left\{c e q_{1}, \ldots, c e q_{n}\right\}, n=5$, be a questionnaire based on the major conceptual CEQ scales with five-point Likert type questions: $c e q_{1}=G T S, c e q_{2}=G S S, c e q_{3}=S S S, c e q_{4}$ $=G Q S$ and $c e q_{5}=O S I$. The objective is to obtain an only questionnaire, based on the output questionnaire type $C E Q$, which integrates the answers to the input questionnaires. 
We propose two steps for solving this integration problem:

(i) A LMCDM process to associate each question of the input questionnaires with a linguistic value indicating the matching of that question with each CEQ scale of the output questionnaire type.

(ii) A LMCDM process to obtain a unique CEQ form type with the integrated answers to the input questionnaires. This integration process will be weighted with the linguistic values obtained in the previous step.

Each of these steps or stages is respectively explained in more details in the following subsections.

\subsection{LMCDM Process to Associate Input Questions with CEQ Scales}

In this decision making process, there are $m$ university education experts $\left\{P_{1}, \ldots, P_{m}\right\}$ who are called to express their performance values on each input question in order to associate them with each CEQ scale of the output questionnaire. Since this is a critical step, we consider that experts should have at least five years of experience. The number of experts should also be at least five, i.e. $m \geq 5$. Experts have to associate each input question to one of the $n$ possible options $(n=5)$ of the available panel. Therefore, each alternative $x_{i}=c e q_{i}$ $\forall i \in\{1, \ldots, n\}$. Finally, experts must also provide their levels of expertise in this particular matching process. These values will be taken into account when weighing their matching ratings. Let $C=\left\{c_{1}, \ldots, c_{m}\right\}$ be the set of experts' expertise levels. Below, we will show how to model this MCDM problem following an indirect linguistic approach.

\subsubsection{Choosing the Linguistic Term Set with its Semantics}

The five-point Likert scale is used for the input and output questionnaires of our system. Therefore, it seems logical that the experts also express all their opinions using this scale. The five-point Likert scale is a set of items made up of an equal number of favorable and unfavorable statements concerning the attitude object. It is provided to a group of subjects that are instructed to select one of five possible responses: "strongly agree", "agree", "neutral", "disagree", or "strongly disagree".

Consequently, we consider the second possibility shown in Section 2.1, which defines the linguistic expression domain by means of an ordered set of linguistic terms. Then, we characterize the linguistic expression domain as follows:

- The granularity value is 5 .

- We consider a linguistic term set on which a total order is defined and distributed on the scale [0, 1], with the mid term representing an assessment of "approximately 0.5 ", with the rest of the terms being placed symmetrically around it.

- We define the semantics by considering that each linguistic term for the pair $\left(s_{i}, s_{T-i}\right)(T+1$ is the cardinality, i.e. 5) is equally informative and by assigning triangular membership functions to each linguistic term.

- Furthermore, we assume three operators, called negation, maximization and minimization, which are respectively defined in $S$ as follows:

$$
\begin{gathered}
\operatorname{neg}\left(s_{i}\right)=s_{j}, \text { with } j=T-i, \\
\max \left(s_{i}, s_{j}\right)=s_{i}, \text { if } s_{i} \geq s_{j}, \text { and } \\
\min \left(s_{i}, s_{j}\right)=s_{i}, \text { if } s_{i} \leq s_{j} .
\end{gathered}
$$

Thus, we can use the set of five linguistic terms shown in Fig 1:

$$
\begin{aligned}
& S=\left\{s_{0}, \ldots, s_{T}\right\}, T=4: s_{0}=\text { Strongly Disagree }=S D, \\
& s_{1}=\text { Disagree }=D, s_{2}=\text { Neutral }=N, s_{3}=\text { Agree }=A, \\
& \text { and } s_{4}=\text { Strongly Agree }=S A .
\end{aligned}
$$

Also, it is possible that the experts do not provide any values, which we symbolize with “-”, in order to express that there is no association with any CEQ scale of the output questionnaire for the input question. Specification of these non-values is a common practice when expressing preferences with linguistic terms.

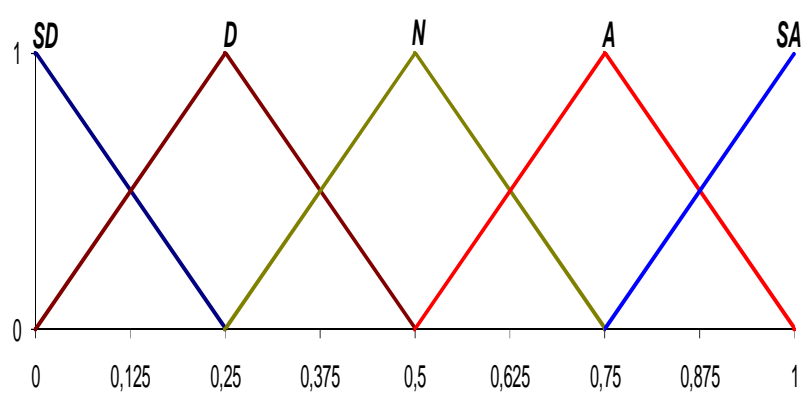

Fig 1. Linguistic terms defined for a five-point Likert scale.

\subsubsection{Choosing the Linguistic Term Set with its Semantics}

An aggregation operator of weighted ordinal fuzzy linguistic information, in particular, the LWA operator 
$\Phi$ presented in Section 2.2.2, is used to aggregate the individual linguistic performance values. This operator is guided by a fuzzy linguistic quantifier, $Q$, representing the concept of fuzzy majority. ${ }^{32}$ In Ref. 43, Yager considers the parameterized family of quantifiers $Q(r)=r^{a}, a \geq 0$ to represent the fuzzy linguistic quantifier most of. Therefore, we propose to use the linguistic quantifier most of defined as $Q(r)=r^{1 / 2}$, $r \in[0,1]$ (see Fig 2). The basic idea consists of using the values $c_{k} \in S$ as the linguistic importance degrees of all the linguistic matching ratings of each expert $P_{k}$.

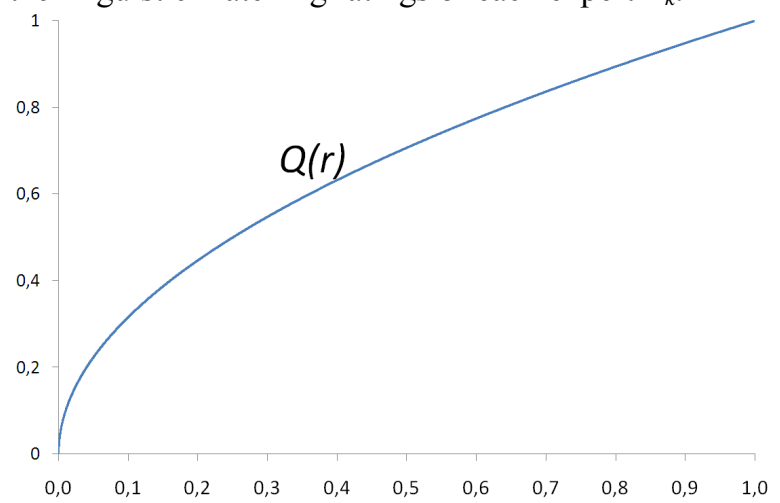

Fig 2. Linguistic quantifier most of: $Q(r)=r^{1 / 2}$.

\subsubsection{Choosing the Best Alternatives}

For each criterion, we assume that linguistic performance values are provided by means of linguistic utility functions. In this case, for each criterion, a utility function is provided for each question of the different input questionnaires:

$$
\begin{aligned}
& V_{k}^{a b}=\left[v_{l}^{k a b}, \ldots, v_{n}^{k a b}\right], \forall k \in\{1, \ldots, m\}, \\
& \forall a \in\{1, \ldots, \# R\}, \forall b \in\left\{1, \ldots, \# R_{a}\right\} .
\end{aligned}
$$

Therefore, each expert $P_{k}$ associates the utility function $V_{k}^{a b}$ for each question $r_{b}{ }^{a}$ with each major conceptual CEQ scale, i.e. the alternative $x_{i}$, by using the linguistic values of $S$ indicating the performance of that alternative.

As was aforementioned in Section 2.3, the proposed choice process is carried out in two phases:

- Aggregation phase of linguistic information. Using the aggregation operator specified above, the collective linguistic utility function $V^{C a b}=\left\{v_{l}^{a b}, \ldots\right.$, $\left.v_{n}{ }^{a b}\right\}$ is obtained for each question $r_{b}{ }^{a}$ from the individual ones $\left\{V_{l}^{a b}, \ldots, V_{m}{ }^{a b}\right\}$ :

$$
V^{C a b}=\left\{\Phi_{Q}\left[\left(c_{1}, v_{1}^{1 a b}\right), \ldots,\left(c_{m}, v_{1}^{m a b}\right)\right], \ldots,\right.
$$

$$
\begin{gathered}
\left.\Phi_{Q}\left[\left(c_{1,} v_{n}{ }^{l a b}\right), \ldots,\left(c_{m}, v_{n}{ }^{m a b}\right)\right]\right\}, \forall a \in\{1, \ldots, \# R\}, \\
\forall b \in\left\{1, \ldots, \# R_{a}\right\}, n=5 .
\end{gathered}
$$

- Exploitation phase for the aggregated linguistic information. The goal of the exploitation phase is to choose the best alternatives from the collective linguistic utility function. Since the linguistic performance values are linguistic utility functions, $V^{C a b}$ is itself a linguistic choice function, i.e. $V^{C a b}=$ $X^{C a b}$. In our problem, we want to obtain for each question of the input questionnaires a linguistic value indicating the matching of that question with each CEQ scale of the output questionnaire. Therefore, this linguistic choice function $X^{C a b}=\left[x_{1}{ }^{a b}, \ldots, x_{n}{ }^{a b}\right]$ is the desired solution, i.e. each $x_{i}^{a b}$ represents the linguistic importance degree of the question $r_{b}{ }^{a}$ for each major conceptual CEQ scale $c e q_{i}$, and these values will be use in the next MCDM step.

\subsection{LMCDM Process to Obtain a CEQ Form from Answers to Input Questionnaires}

Assuming that we have several groups of students (decision makers), $Y_{a}=\left\{y_{1}^{a}, \ldots, y_{\# Y a}{ }^{a}\right\}, \# Y_{a} \geq 1$, which have filled in the corresponding questionnaires $R_{a}$, we consider that $e_{d}^{a}\left(r_{b}{ }^{a}\right), \forall d \in\left\{1, \ldots, \# Y_{a}\right\}$, is the opinion provided by the student $e_{d}^{a}$ on subjective criteria represented by the question $r_{b}{ }^{a}$. The objective is to obtain a unique CEQ type form, $C E Q_{o}=\left\{c e q_{1}^{o}, \ldots\right.$, $\left.c e q_{n}{ }^{o}\right\}, n=5$, with the integrated answers to the input questionnaires, i.e. $e_{d}{ }^{a}\left(r_{b}{ }^{a}\right), \forall a \in\{1, \ldots, \# R\}, \forall b \in$ $\left\{1, \ldots, \# R_{a}\right\}, \forall d \in\left\{1, \ldots, \# Y_{a}\right\}$.

Since in this decision making process all the answers to the input questionnaires have to be integrated into a panel with $n$ possible options, we consider that each alternative $x_{i}=c e q_{i}, \forall i \in\{1, \ldots, n\}$. Below, we will explain how to model this MCDM problem following an indirect linguistic approach.

\subsubsection{Choosing the Linguistic Term Set with its Semantics}

As aforementioned, the five-point Likert scale has been used to express users' opinions in all the questionnaires of our system. Therefore, $e_{d}^{a}\left(r_{b}{ }^{a}\right), c e q_{i}^{o} \in S$, where the set $S$ is the same that we have defined for the inputs questionnaires. Furthermore, the considerations on this linguistic expression domain are the same as those already described in Section 4.1.1. 


\subsubsection{Choosing the Linguistic Information Aggregation Operator}

Again, the LWA operator $\Phi$ (presented in Section 2.2.2), which aggregates weighted ordinal fuzzy linguistic information, is used to aggregate the individual linguistic performance values, i.e. the answers to input questionnaires. We also use the linguistic quantifier most of, defined as $Q(r)=r^{1 / 2}$. At the end of the previous MCDM step, we obtained the linguistic choice function $V^{C a b}=X^{C a b}$, see Eq. (4.1). The basic idea consists of using $x_{i}^{a b}$ as the linguistic importance degrees of all the linguistic answers to input questionnaires, i.e. $e_{d}{ }^{a}\left(r_{b}{ }^{a}\right)$, in order to obtain the corresponding output questionnaire value $c e q_{i}{ }^{o}$ for each major conceptual CEQ scale $c e q_{i}$.

\subsubsection{Choosing the Best Alternatives}

Once more, the proposed choice process is carried out in two phases:

- Aggregation phase of linguistic information. For each criterion, we assume that linguistic performance values about the alternatives are provided by means of linguistic utility functions. Using the aggregation operator specified in the previous section, the collective linguistic utility function $V^{C}$ is obtained for each question of the output questionnaire:

$$
\begin{gathered}
V^{C}=\left\{\Phi_{Q}\left[\left(x_{l}^{a b}, e_{d}^{a}\left(r_{b}^{a}\right)\right)\right], \ldots, \Phi_{Q}\left[\left(x_{n}^{a b}, e_{d}^{a}\left(r_{b}^{a}\right)\right)\right]\right\}, \\
\forall a \in\{1, \ldots, \# R\}, \forall b \in\left\{1, \ldots, \# R_{a}\right\}, \\
\forall d \in\left\{1, \ldots, \# Y_{a}\right\}, n=5 .
\end{gathered}
$$

- Exploitation phase for the aggregated linguistic information. Since the linguistic performance values are linguistic utility functions, $V^{C}$ is a linguistic choice function, i.e. $V^{C}=X^{C}$. Therefore, this collective vector is the desired solution, i.e. $V^{C}=C E Q_{o}$.

\section{An Application Example}

Several web questionnaires asking different questions have been used during the last years to survey the students enrolled on ICT courses taught at the Business Studies Faculty of the University of Granada. The new model proposed in this paper has been applied to integrate these heterogeneous questionnaires. The objective has been to aggregate these questionnaires into five-point Likert scale CEQ forms that characterize them on their common features: students of ICT courses at the Business Studies Faculty of the University of
Granada, and the corresponding academic year. In this section, we present a limited example, where the model is applied to integrate one of these questionnaires using an ICT laboratory group of students in the academic year 2009-10.

Let $R=\left\{R_{l}\right\}$ be the set of input questionnaires, which only contains the example questionnaire $R_{l}$ shown in Fig 3, and let $R_{1}=\left\{r_{1}{ }^{l}, \ldots, r_{11}{ }^{l}\right\}$ be the set of 11 five-point Likert-type questions of such questionnaire. The objective is to obtain a unique output questionnaire (of type CEQ), which integrates the answers to the input questionnaire.

As aforementioned, we have to carry out two steps for solving this integration problem:

(i) A LMCDM process to associate each question of the input questionnaire with a linguistic value indicating the matching of that question with each CEQ scale of the output questionnaire. This step is solved using the LMCDM process proposed in Section 4.1.

(ii) A LMCDM process to obtain a unique CEQ type form with the integrated answers to the input questionnaire. This step is solved using the LMCDM process proposed in Section 4.2.

In the following subsections, we will explain these steps in more details.

\subsection{Example of the LMCDM Process to Associate Input Questions with CEQ Scales}

In this decision making process, there are 5 university education experts:

- $P_{1}$ : Full Professor with more than 20 years of experience.

- $P_{2}$ : Assistant professor with more than 15 years of experience.

- $P_{3}$ : Assistant professor with more than 12 years of experience.

- $P_{4}$ : Assistant professor with more than 10 years of experience.

- $P_{5}$ : Associate Professor with 7 years of experience.

They are called to express their linguistic performance values on each input question in order to associate them with each CEQ scale of the output questionnaire. Let us remember that it is possible that experts do not provide any values (which is coded as "-") to some questions. Besides, these experts present the following set $C$ evaluating their levels of expertise in this particular matching process:

$$
C=\{S A, S A, S A, S A, A\}
$$




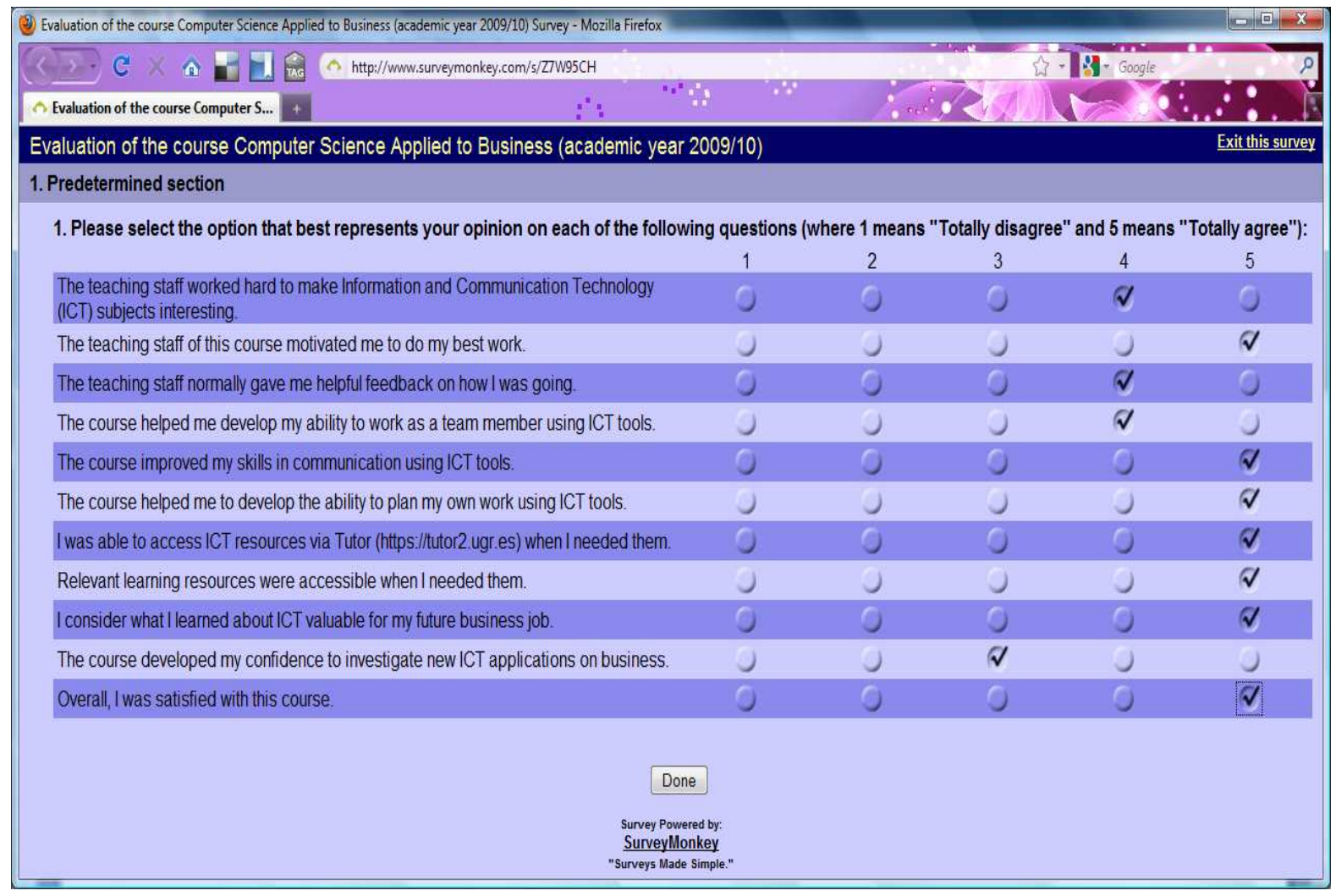

Fig 3. Example of questionnaire $\left(R_{l}\right)$.

For example, for the question number $10, r_{10}{ }^{1}$, the linguistic utility functions provided are:

$$
\begin{aligned}
& V_{1}^{110}=[N, A, D, S A, N], V_{2}^{110}=[N, S A, D, S A, D], \\
& V_{3}^{110}=[N, A, S D, S A, N], V_{4}^{110}=[N, A, S D, S A, N], \\
& V_{5}^{1{ }^{10}}=[S D, S A, S D, S A, D] .
\end{aligned}
$$

Using Eq. (4.1), we obtain the following collective linguistic preference relation, i.e. the linguistic choice function:

$$
\begin{aligned}
& V^{C} 110=X^{C} 110 \\
& =\left\{\Phi_{Q}[(S A, N),(S A, N),(S A, N),(S A, N),(A, S D)],\right. \\
& \Phi_{Q}[(S A, A),(S A, S A),(S A, A),(S A, A),(A, S A)], \\
& \Phi_{Q}[(S A, D),(S A, D),(S A, S D),(S A, S D),(A, S D)], \\
& \Phi_{Q}[(S A, S A),(S A, S A),(S A, S A),(S A, S A),(A, S A)],
\end{aligned}
$$

$$
\begin{gathered}
\left.\Phi_{Q}[(S A, N),(S A, D),(S A, N),(S A, N),(A, D)]\right\} \\
=\{N, A, D, S A, N\}
\end{gathered}
$$

For instance, the assessment for the GSS scale (the second CEQ scale) from $r_{10}{ }^{l}$ is obtained using the following expression:

$$
\Phi_{Q}[(S A, A),(S A, S A),(S A, A),(S A, A),(A, S A)]=A
$$

To develop this expression is necessary to calculate the weighting vector $W$. To do so, we make use of the linguistic quantifier most of specified in Section 4.1.2, and we obtain the following weighting vector, using Eq. (2.2):

$$
W=(0.45,0.19,0.14,0.12,0.11)
$$

Using Eq. (2.1) orness $(W)=0.69$. As this value is greater than 0.5 , then $h=\min \left(c_{i}, a_{i}\right)$, and therefore:

$$
\Phi_{Q}[(S A, A),(S A, S A),(S A, A),(S A, A),(A, S A)]
$$




$$
\begin{gathered}
=\varphi_{Q}(A, S A, A, A, A)=W \cdot B^{T} \\
=C^{5}\{(A, 0.45),(S A, 0.19),(A, 0.14),(A, 0.12), \\
(A, 0.11)\} \\
=0.45 \otimes A \oplus 0.55 \otimes C^{4}\{(S A, 0.34),(A, 0.26), \\
(A, 0.22),(A, 0.19)\}=A
\end{gathered}
$$

Using the same LMCDM process for the remaining

\begin{tabular}{|c|c|c|c|c|c|c|}
\hline$r_{b}{ }^{l}$ & Question & $\begin{array}{l}x_{1}{ }^{l b} \\
G T S\end{array}$ & $\begin{array}{l}x_{2}{ }^{l b} \\
G S S\end{array}$ & $\begin{array}{l}x_{3}{ }^{l b} \\
S S S\end{array}$ & $\begin{array}{l}x_{4}^{l b} \\
G Q S\end{array}$ & $\begin{array}{l}x_{5}^{I b} \\
\text { OSI }\end{array}$ \\
\hline$r_{l}^{l}$ & $\begin{array}{l}\text { The teaching staff worked } \\
\text { hard to make Information } \\
\text { and Communication } \\
\text { Technology (ICT) subjects } \\
\text { interesting. }\end{array}$ & $S A$ & - & - & $A$ & $S A$ \\
\hline$r_{2}^{l}$ & $\begin{array}{l}\text { The teaching staff of this } \\
\text { course motivated me to do } \\
\text { my best work. }\end{array}$ & $S A$ & - & - & $N$ & $S A$ \\
\hline$r_{3}{ }^{l}$ & $\begin{array}{l}\text { The teaching staff normally } \\
\text { gave me helpful feedback } \\
\text { on how I was going. }\end{array}$ & $S A$ & - & $A$ & - & $S A$ \\
\hline$r_{4}^{l}$ & $\begin{array}{l}\text { The course helped me } \\
\text { develop my ability to work } \\
\text { as a team member using } \\
\text { ICT tools. }\end{array}$ & $A$ & $S A$ & - & $S D$ & $S D$ \\
\hline$r_{5}{ }^{1}$ & $\begin{array}{l}\text { The course improved my } \\
\text { skills in communication } \\
\text { using ICT tools. }\end{array}$ & $N$ & $S A$ & - & $A$ & $S D$ \\
\hline$r_{6}{ }^{l}$ & $\begin{array}{l}\text { The course helped me to } \\
\text { develop the ability to plan } \\
\text { my own work using ICT } \\
\text { tools. }\end{array}$ & $N$ & $S A$ & - & $A$ & $D$ \\
\hline$r_{7}^{l}$ & $\begin{array}{l}\text { I was able to access ICT } \\
\text { resources via Tutor } \\
\text { (https://tutor2.ugr.es) when } \\
\text { I needed them. }\end{array}$ & - & - & $S A$ & - & - \\
\hline$r_{8}{ }^{l}$ & $\begin{array}{l}\text { Relevant learning resources } \\
\text { were accessible when I } \\
\text { needed them. }\end{array}$ & - & - & $S A$ & - & $S D$ \\
\hline$r_{9}^{l}$ & $\begin{array}{l}\text { I consider what I learned } \\
\text { about ICT valuable for my } \\
\text { future business job. }\end{array}$ & $D$ & $A$ & - & $S A$ & $A$ \\
\hline$r_{10}{ }^{l}$ & $\begin{array}{l}\text { The course developed my } \\
\text { confidence to investigate } \\
\text { new ICT applications on } \\
\text { business. }\end{array}$ & $N$ & $A$ & $D$ & $S A$ & $N$ \\
\hline$r_{11}^{I}$ & $\begin{array}{l}\text { Overall, I was satisfied with } \\
\text { this course. }\end{array}$ & $A$ & $N$ & $N$ & $D$ & $S A$ \\
\hline
\end{tabular}
questions, i.e. applying Eq. (4.1) to each input question, we obtain the result shown in Table 2 .

Table 2. Result of the LMCDM process to associate the input questions of $R_{l}$ with the CEQ scales $\left(X^{C a b}\right)$.

\subsection{Example of the LMCDM Process to Get a CEQ Form from Answers to Input Questionnaires}

We have a group of ten students $Y_{1}=\left\{y_{1}{ }^{l}, \ldots, y_{10}{ }^{l}\right\}$ who have filled in the questionnaire $R_{l}$. Table 3 shows all the opinions provided by the students answering this

\begin{tabular}{|c|c|c|c|c|c|c|c|c|c|c|c|}
\hline \multirow{2}{*}{$\begin{array}{c}\text { student } \\
\qquad y_{d}^{l}\end{array}$} & \multicolumn{11}{|c|}{ answers: $e_{d}^{l}\left(r_{b}{ }^{l}\right)$} \\
\hline & $r_{1}^{I}$ & $r_{2}^{l}$ & $r_{3}^{I}$ & $r_{4}^{1}$ & $r_{5}^{1}$ & $r_{6}^{l}$ & $r_{7}^{I}$ & $r_{8}^{l}$ & $r_{9}^{l}$ & $r_{10}^{1}$ & $r_{11}^{l}$ \\
\hline$y_{1}^{l}$ & $S A$ & $A$ & $A$ & $S A$ & $A$ & $A$ & $S A$ & $A$ & $S A$ & $S A$ & $S A$ \\
\hline$y_{2}{ }^{l}$ & $A$ & $A$ & $S A$ & $N$ & $A$ & $N$ & $S A$ & $A$ & $S A$ & $S A$ & $A$ \\
\hline$y_{3}{ }^{l}$ & $S A$ & $S A$ & $S A$ & $S A$ & $S A$ & $A$ & $A$ & $D$ & $N$ & $N$ & $S A$ \\
\hline$y_{4}{ }^{I}$ & $D$ & $S A$ & $S A$ & $S A$ & $S A$ & $A$ & $S A$ & $A$ & $A$ & $D$ & $S A$ \\
\hline$y_{5}{ }^{l}$ & $A$ & $S A$ & $S A$ & $S A$ & $S A$ & $N$ & $S A$ & $N$ & $S A$ & $S A$ & $S A$ \\
\hline$y_{6}{ }^{l}$ & $S A$ & $S A$ & $S A$ & $S A$ & $A$ & $N$ & $S A$ & $N$ & $A$ & $N$ & $A$ \\
\hline$y_{7}^{l}$ & $N$ & $N$ & $D$ & $S D$ & $S D$ & $S D$ & $A$ & $N$ & $S D$ & $S D$ & $N$ \\
\hline$y_{8}{ }^{l}$ & $S A$ & $S A$ & $S A$ & $S A$ & $S A$ & $S A$ & $S A$ & $A$ & $A$ & $S A$ & $S A$ \\
\hline$y_{9}{ }^{l}$ & $A$ & $S A$ & $S A$ & $S A$ & $S A$ & $S A$ & $S A$ & $S A$ & $N$ & $A$ & $A$ \\
\hline$y_{10}{ }^{l}$ & $S A$ & $S A$ & $S A$ & $S A$ & $A$ & $A$ & $S A$ & $A$ & $S A$ & $S A$ & $S A$ \\
\hline
\end{tabular}
questionnaire. The objective is to obtain a unique CEQ type form with the integrated answers to this input questionnaire.

Table 3. Students' answers to the questionnaire $R_{l}$.

In order to get the final solution in this decision making process, we use the linguistic choice functions $x_{i}^{l b}$ obtained in the previous LMCDM step (shown in Table 2) as the linguistic importance degrees of all the linguistic answers to each question $r_{b}{ }^{l}$ of the input questionnaire, i.e. we apply Eq. (4.2) as follows:

$$
\begin{aligned}
& V^{C}=X^{C}=C E Q_{o} \\
& =\left\{\Phi_{Q}\left[\left(x_{1}{ }^{l b}, e_{d}^{l}\left(r_{b}^{l}\right)\right)\right], \Phi_{Q}\left[\left(x_{2}{ }^{l b}, e_{d}^{l}\left(r_{b}^{l}\right)\right)\right],\right. \\
& \Phi_{Q}\left[\left(x_{3}{ }^{l b}, e_{d}^{l}\left(r_{b}{ }^{l}\right)\right)\right], \Phi_{Q}\left[\left(x_{4}{ }^{l b}, e_{d}^{l}\left(r_{b}{ }^{l}\right)\right)\right], \\
& \left.\Phi_{Q}\left[\left(x_{5}{ }^{l l}, e_{1}^{l}\left(r_{1}^{l}\right)\right)\right]\right\}=\{S A, A, A, A, S A\}
\end{aligned}
$$

Thus, according to our model, we can say that students who filled out the questionnaire $R_{1}$ are strongly agree on the Overall Satisfaction Index and the Good Teaching Scale and agree on the rest of the CEQ scales.

For instance, the integrated answer to the SSS (the third CEQ scale) scale is obtained from the following expression:

$$
\begin{array}{cl}
\Phi_{Q}\left[\left(x_{3}{ }^{l b}, e_{d}{ }^{l}\left(r_{b}{ }^{l}\right)\right)\right] & \\
=\Phi_{Q}[(A, A), \quad(S A, S A), & (S A, A), \quad(D, S A), \quad(N, S A), \\
(A, S A),(S A, S A), & (S A, A),(D, S A), \quad(N, A), \\
(A, S A), \quad(S A, A), & (S A, D), \quad(D, N),(N, S A),
\end{array}
$$


$(A, S A), \quad(S A, S A), \quad(S A, A),(D, D), \quad(N, S A)$, $(A, S A), \quad(S A, S A),(S A, N), \quad(D, S A), \quad(N, S A)$, $(A, S A), \quad(S A, S A), \quad(S A, N), \quad(D, N), \quad(N, A)$, $(A, D), \quad(S A, A), \quad(S A, N), \quad(D, S D), \quad(N, N)$, $(A, S A), \quad(S A, S A), \quad(S A, A),(D, S A), \quad(N, S A)$, $(A, S A), \quad(S A, S A), \quad(S A, S A), \quad(D, A), \quad(N, A)$, $(A, S A),(S A, S A),(S A, A), \quad(D, S A), \quad(N, S A)]$ $=A$.

\section{Concluding Remarks}

We have presented a model made up of two LMCDM processes for integrating the answers to very heterogeneous university-level questionnaires, in order to obtain a unique questionnaire based on the CEQ form. The aim of the first process is to associate each question of the input questionnaires with each one of the output questionnaire, according to the consensus of a panel of university education experts. The goal of the second process is to obtain a unique output questionnaire with the integrated answers to the input questionnaires, according to the students who have filled in the corresponding input questionnaires and the results of the previous LMCDM step. The main advantage of having this integrated questionnaire is that its results can be incorporated into other CEQ surveys to make benchmarking among organizations. All the questionnaires involved in our system (input questionnaires and the output CEQ form) are based on a five-point Likert scale. We are currently focusing on the management of questionnaires based on other domains, including natural language (open-ended questions). Besides, we are working on extending the solution proposed here to similar integration problems, in particular, to reputational risk management ones.

\section{Acknowledgments}

This paper has been developed with the financing of FEDER funds in FUZZYLING-II Project TIN201017876 and Andalusian Excellence Projects TIC-05299 and TIC-5991.

\section{References}

1. C. McInnis, Studies of Student Life: an overview, European Journal of Education 39(4) (2004) 383-394.

2. G. D. Kuh, The National Survey of Student Engagement: Conceptual Framework and Overview of Psychometric Properties (2003) Indiana University Center for Postsecondary Research and Planning, http://nsse.iub.edu/pdf/conceptual_framework_2003.pdf.
3. P. Ramsden and N. J. Entwistle, Effects of Academic Departments on Students' Approaches to Studying. British Journal of Educational Psychology 51 (1981) 368-383.

4. P. Ramsden, E. Martin and J. Bowden, School environment and sixth form pupil's approaches to learning. British Journal of Educational Psychology 59 (1989) 129-142.

5. C. McInnis, P. Griffin, R. James and H. Coates, Development of the Course Experience (DETYA, Canberra, 2001).

6. K. Wilson, A. Lizzio and P. Ramsden, The Development, Validation and Application of the Course Experience Questionnaire. Studies in Higher Education 1(1997) 3353 .

7. J. A. Gliem, R. R. Gliem, Calculating, Interpreting, and Reporting Cronbach's Alpha Reliability Coefficient for Likert-Type Scales, Midwest Research to Practice Conference in Adult, Continuing, and Community Education, (The Ohio State University, Columbus, 2003), pp. 82-88.

8. R. Likert. A technique for the measurement of attitudes. Archives of Psychology (Columbia University Press, New York, 1931).

9. J. P. McIver and E. G. Carmines, Unidimensional scaling (Sage, Newbury Park, CA, 1981).

10. W. Deng and W. Pei, Fuzzy neural based importanceperformance analysis for determining critical service attributes. Expert Syst. Appl. 36(2) (2009) 3774-3784.

11. H. K. Chiou and G. H. Tzeng and D. C. Cheng, Evaluating sustainable fishing development strategies using fuzzy MCDM approach. Omega 33 (2005) 223234.

12. L. A. Zadeh, Fuzzy sets. Information and Control 8 (1965) 338-353.

13. L. A. Zadeh, The concept of a linguistic variable and its applications to approximate reasoning, Inf. Sci. 8(pt. I, II) (1975) 199-249 and 301-357.

14. F. Chiclana, F. Herrera and E. Herrera-Viedma, Integrating three representation models in fuzzy multipurpose decision making based on fuzzy preference relations, Fuzzy Sets and Systems 97 (1998) 33-48.

15. F. Herrera, E. Herrera-Viedma and L. Martinez, A fusion approach for managing multi-granularity linguistic term sets in decision making, Fuzzy Sets and Systems 114 (2000) 43-58.

16. F. Herrera and E. Herrera-Viedma, Linguistic decision analysis: Steps for solving decision problems under linguistic information, Fuzzy Sets and Systems 115(10) (2000) 67-82.

17. G. Bordogna and G. Passi, A fuzzy linguistic approach generalizing Boolean information retrieval: A model and its evaluation, J. Amer. Soc. Inf. Sci. 44 (1993) 70-82.

18. E. Herrera-Viedma, A. G. López-Herrera, A Review on Information Accessing Systems Based on Fuzzy Linguistic Modelling. International Journal of Computational Intelligence Systems 3(4) (2010) 420-437 
19. E. Herrera-Viedma, An information retrieval system with ordinal linguistic weighted queries based on two weighting elements, Int. J. Uncertainty, Fuzziness Knowl. Based Syst. 9 (2001) 77-88.

20. E. Herrera-Viedma, A. G. López-Herrera, M. Luque and C. Porcel, A fuzzy linguistic IRS model based on a 2tuple fuzzy linguistic approach, Int. J. Uncertainty, Fuzziness Knowl. Based Syst. 15 (2007) 225-250.

21. M. Delgado, J. L. Verdegay and M. A. Vila, Linguistic decision making models, Int. J. Intell. Syst. 7 (1992) 479 492.

22. F. Mata, L. Martínez, E. Herrera-Viedma, An Adaptive Consensus Support Model for Group Decision Making Problems in a Multi-Granular Fuzzy Linguistic Context. IEEE Transactions on Fuzzy Systems 17(2) (2009) 279290.

23. J. L. Garcia-Lapresta, B. Llamazares and M. MartinezPanero. A Social Choice Analysis of the Borda Rule in a General Linguistic Framework, International Journal of Computational Intelligence Systems 3(4) (2010) 501513.

24. P. P. Bonissone and K. S. Decker, Selecting Uncertainty Calculi and Granularity: An Experiment in Trading-off Precision and Complexity, Uncertainty in Artificial Intelligence, eds. L. H. Kanal and J. F. Lemmer, (NorthHolland, Amsterdam, 1986), pp. 217-247.

25. P. P. Bonissone, A fuzzy sets based linguistic approach: Theory and applications, Approximate Reasoning in Decision Analysis eds. M. M. Gupta and E. Sanchez (North-Holland, Amsterdam, 1982), pp. 329-339.

26. F. Herrera, E. Herrera-Viedma, and J. L. Verdegay, A sequential selection process in group decision making with linguistic assessment, Inf. Sci. 85 (1995) 223-239.

27. R. R. Yager, An approach to ordinal decision making, Int. J. Approx. Reas. 12 (1995) 237-261.

28. B. Bouchon-Meunier and J. Yao, Linguistic Modifiers and imprecise categories, Int. J. Intell. Syst. 7 (1992) 2536.

29. M. Delgado, J. L. Verdegay and M. A. Vila, On aggregation operations of linguistic labels, Int. J. Intell. Syst. 8 (1993) 351-370.

30. F. Herrera, E. Herrera-Viedma and J. L. Verdegay, Direct approach processes in group decision making using linguistic OWA operators, Fuzzy Sets and Systems 79 (1996) 175-190.

31. F. Herrera and E. Herrera-Viedma, Aggregation operators for linguistic weighted information, IEEE Transactions on Systems, Man and Cybernetics, Part A: Systems and Humans 27(5) (1997) 646-656.

32. R. R. Yager, On ordered weighted averaging aggregation operators in multicriteria decision making, IEEE Transactions on Systems, Man and Cybernetics 18(1) (1988) 183-190.

33. J. Kacprzyk, Group decision making with a fuzzy linguistic majority, Fuzzy Sets Systems 18(2) (1986) 105118.
34. L. A. Zadeh, A computational approach to fuzzy quantifiers in natural languages, Computer \& Mathematics with Applications 9(1) (1983) 149-184.

35. F. Herrera, E. Herrera-Viedma and J.L. Verdegay, Choice processes for non-homogeneous group decision making in linguistic setting, Fuzzy Sets and Systems 94 (1997) 287-308.

36. F. Herrera, E. Herrera-Viedma and J.L. Verdegay, A rational consensus model in group decision making using linguistic assessments, Fuzzy Sets and Systems 88 (1997) 31-49.

37. G. Bordogna, M. Fedrizzi and G. Passi, A linguistic modelling of consensus in group decision making based on OWA operators, IEEE Trans. Systems Man Cybernet. 27 (1997) 126-132.

38. M. Fedrizzi and L. Mich, Rule based model for consensus reaching group decisions support, Proc. 3rd Conf. on Information Processing and Management of Uncertainty, (Palma de Mallorca, 1992), pp. 301-304.

39. R. R. Yager, Non-numeric multi-criteria multi-person decision making, Group Decision Negotiation 2 (1993) 81-93.

40. S. A. Orlovsky, Decision making with a fuzzy preference relation, Fuzzy Sets and Systems 1 (1978) 155-167.

41. M. Roubens, Some properties of choice functions based on valued binary relations, European Journal of Operational Research 40 (1989) 309-321.

42. F. Herrera and E. Herrera-Viedma, Choice Functions and Mechanisms for Linguistic Preference Relations, European Journal of Operational Research 120 (2000) 144-161.

43. R. R. Yager, Quantifier guided aggregation using OWA operators, Int. J. Intell. Syst. 11(1) (1996) 49-73. 